\title{
Projeto ESPAÇOS de Curitiba, Brasil: aplicabilidade de métodos mistos de pesquisa e informações georreferenciadas em estudos sobre atividade física e ambiente construído
}

\author{
Adriano Akira Ferreira Hino, ${ }^{1}$ Cassiano Ricardo Rech, ${ }^{2}$ \\ Priscila Bezerra Gonçalves, ${ }^{3}$ Pedro Curi Hallal ${ }^{4}$ e Rodrigo Siqueira Reis ${ }^{3}$
}

Como citar Hino AAF, Rech CR, Gonçalves PB, Hallal PC, Reis RS. Projeto ESPAÇOS de Curitiba, Brasil: aplicabilidade de métodos mistos de pesquisa e informações georreferenciadas em estudos sobre atividade física e ambiente construído. Rev Panam Salud Publica. 2012;32(3):226-33.

RESUMO Objetivo. Descrever os métodos empregados para avaliar o ambiente construído e a atividade física em um estudo multicêntrico internacional e discutir os desafios enfrentados para obter os dados necessários no contexto brasileiro.

Métodos. Em 2010, foi conduzido um inquérito domiciliar com adultos entre 20 e 65 anos de idade na Cidade de Curitiba, Estado do Paraná. O estudo envolveu o uso de informações georreferenciadas para identificar locais que facilitem a caminhada como forma de deslocamento (walkability) em todos os 2125 setores censitários de Curitiba. Os setores censitários foram categorizados de acordo com walkability e renda, a qual considerou a renda média dos responsáveis pelos domicílios contidos em cada setor. A atividade física foi avaliada de maneira autorrelatada e objetiva (acelerômetro).

Resultados. Foram incluídos 16 setores de alto walkability e 16 de baixo walkability, sendo oito de baixa renda e oito de renda elevada em cada categoria. Foram entrevistados 699 sujeitos e 381 utilizaram acelerômetros. A taxa de respostas para as entrevistas foi de 66,4\%. O sucesso no uso de acelerômetros foi de $85,8 \%(n=327)$.

Conclusões. Os resultados demonstram que é possível desenvolver estudos de elevada qualidade sobre AF e ambiente construído no contexto brasileiro, seguindo padrões internacionais de investigação.

Palavras-chave Atividade motora; planejamento ambiental; desenho experimental; Brasil.

1 Universidade Federal do Paraná (UFPR), Programa de Pós Graduação em Educação Física, Curitiba (PR) Brasil. Correspondência: akira_manaca@yahoo.com.br

2 Universidade Estadual de Ponta Grossa (UEPG), Ponta Grossa (PR), Brasil.

3 Pontifícia Universidade Católica do Paraná (PUCPR), Escola de Saúde e Biociências, Grupo de Pesquisa em Atividade Física e Qualidade de Vida (GPAQ), Curitiba (PR), Brasil.

4 Universidade Federal de Pelotas (UFPel), Pelotas (RS), Brasil.
Inúmeros fatores contribuem para a inatividade física, quarta causa de morte no mundo (1). Entre esses fatores, são os aspectos do ambiente que têm recebido, nos últimos anos, atenção especial em diversos países, inclusive na América Latina $(2,3)$. Nessa região, destaca-se o impacto da rápida urbanização sobre as doenças crônicas não transmissíveis (4).
Os atributos ambientais que podem influenciar a atividade física (AF) são classificados em construídos ou naturais (5). O ambiente construído caracteriza-se por estruturas e espaços modificados diretamente pelo ser humano, como edifícios, sistemas viários, praças e outros (5). Esses atributos, predominantes nos centros urbanos, juntamente com seu 
potencial impacto nos comportamentos da população, têm motivado a investigação acerca da relação entre ambiente construído e AF.

Há evidências de que as pessoas que vivem em locais com uso diversificado do solo, ou seja, em áreas que comportam tanto residências quanto estabelecimentos comerciais, educacionais, governamentais e espaços públicos, com maior densidade residencial e maior conectividade de ruas, caminham e pedalam mais em seus deslocamentos $(6,7)$. Da mesma maneira, calçadas bem conservadas, beleza arquitetônica, disponibilidade de equipamentos para $\mathrm{AF}$, presença de pistas para caminhada, ciclovias e parques são associados a maior prática de AF no lazer $(7,8)$. No entanto, a quase totalidade dessas evidências é oriunda de um número pequeno de cidades de países de renda alta $(7,8)$. Assim, pouco se conhece sobre o quanto a baixa variabilidade de características do ambiente construído (variável de exposição) pode subestimar a associação do ambiente construído com a AF (9). Também não se sabe até que ponto as evidências disponíveis podem ser extrapoladas para locais de renda média, como os países da América Latina, inclusive o Brasil. Tal aspecto assume grande relevância, considerando que mais de $85 \%$ da população na América Latina reside atualmente em áreas urbana, especialmente em cidades com mais de 1 milhão de habitantes (10).

As lacunas de conhecimento acerca da relação entre ambiente construído e AF motivaram o desenvolvimento de uma investigação que possibilitasse: a) a identificação de oportunidades para aumentar a variabilidade de características do ambiente construído entre países e dentro de cada país; b) a aplicação de métodos comuns e aceitos internacionalmente, que possibilitem a comparação dos resultados entre os estudos e análises conjuntas entre diferentes localidades e países; c) a construção de medidas objetivas de ambiente e de AF. Nessa perspectiva, foi proposto, em 2009, o Estudo Internacional de Atividade Física e Ambiente (International Physical Activity and Environment Study, IPENStudy), conduzido em diversas regiões do mundo.

No IPEN-Study, os atributos do ambiente com potencial impacto sobre a AF realizada nos deslocamentos diários são operacionalizados e representados pelo termo walkability. Esta palavra, que não guarda similar na língua portuguesa, tem sido empregada para descrever locais que facilitem o deslocamento a pé e pode ser operacionalizada pela combinação de ao menos três variáveis: uso diversificado do solo (combinação de áreas residenciais e comerciais), alta densidade residencial e conectividade das ruas (5).

Os objetivos deste artigo são descrever os métodos empregados pelo IPENStudy no Brasil para avaliar o ambiente construído e a AF e discutir os desafios enfrentados na aplicação desses métodos no contexto de um país de renda média.

\section{MATERIAIS E MÉTODOS}

O IPEN-Study é um estudo epidemiológico observacional transversal internacional. No Brasil, foi realizado na Cidade de Curitiba (Paraná). A cidade é internacionalmente reconhecida pela grande disponibilidade de áreas verdes, parques e praças e também por seu sistema de transporte diferenciado e inovador (11). Em Curitiba, o longo histórico de planejamento urbano possibilitou o acúmulo de dados para estudos na área de ambiente, inclusive pela existência do Instituto de Planejamento Urbano, ou IPPUC (12), o qual conta com um vasto acervo de informações georreferenciadas. Finalmente, a experiência do Grupo de Pesquisa em Atividade Física e Qualidade de Vida (GPAQ) em estudos sobre ambiente e AF (12-14) e com diferentes métodos aplicados a esta temática $(15,16)$ representou uma combinação decisiva para o desenvolvimento do estudo.

O emprego de um nome na língua inglesa e o caráter emergente da temática representaram uma barreira potencial para o engajamento da comunidade. Por essa razão, adotou-se um nome "local" para o estudo: Projeto E.S.P.A.C.O.S. de Curitiba - Entendimento Sobre a Prática de Atividade Física nas Comunidades.

\section{Características do IPEN-Study}

O IPEN-Study tem como objetivo avaliar os efeitos do ambiente construído e da renda sobre a AF. Para tanto, as cidades participantes do estudo adotam uma estratégia comum: a) identificar áreas que ofereçam maior ou menor potencial para $\mathrm{AF}$; b) identificar áreas com maior ou menor renda; c) identificar áreas que combinem as duas características.
A seleção dos locais e participantes tem como premissa o papel do ambiente construído nos padrões de AF das pessoas e comunidades (17). A definição operacional de walkability empregada neste estudo já foi anteriormente utilizada $(18,19)$.

\section{Unidades amostrais primárias}

As unidades primárias de amostragem (UPA) do IPEN-Study são definidas a partir das características de walkability de uma determinada área. Em Curitiba, optou-se por utilizar os setores censitários como UPA. O setor censitário é definido como uma área contínua, com dimensão e número de domicílios que permitam levantamento das informações por um único agente do censo. De acordo com o censo de 2000, Curitiba é composta por 2125 setores censitários, cada um com área média de $0,21 \mathrm{~km}^{2}$ e 225 domicílios.

\section{Desenvolvimento do escore de walkability}

Neste estudo, foram empregados quatro indicadores para compor o escore de walkability: densidade residencial, conectividade das ruas, uso diversificado do solo e densidade comercial. Para tanto, foram utilizadas informações baseadas no sistema de informações geográficas (SIG) disponibilizados pelo IPPUC.

A densidade residencial foi estimada a partir de dados do Censo 2000, considerando a razão entre o número de domicílios e a área de cada setor censitário. A conectividade das ruas foi determinada pelo número de intersecções formadas por quatro ou mais segmentos de ruas por área de cada setor. Como as intersecções localizadas no limite entre os setores não poderiam estar contidas em dois ou mais setores, descaracterizando dessa forma o número de segmentos de ruas e correndo o risco de ser excluídas da contagem, um raio adicional de 10 metros ao longo do perímetro de cada setor foi traçado para incluir as intersecções de ruas contidas nesse espaço.

$\mathrm{O}$ uso diversificado do solo foi determinado pelo cálculo da entropia. A entropia varia de 0 (predominância de apenas um tipo de uso do solo) a 1 (distribuição igual entre todas as categorias de uso). As informações sobre os lotes da cidade foram agrupadas em cinco categorias de uso: residencial, comercial, re- 
creativo, educacional/cultural e outros. $\mathrm{O}$ indicador foi calculado pela equação:

$$
\text { Entropia }=-\frac{\sum k\left(\mathrm{p}_{k} 1 n \mathrm{p}_{k}\right)}{1 n N}
$$

onde p é a proporção do uso de solo; ln é o logaritmo natural; $\mathrm{N}$ é o número de categorias do uso de solo; e k é a categoria do uso de solo - 1) residencial, 2) $\mathrm{CO}^{-}$ mercial, 3) recreativo, 4) educacional/ cultural e 5) outros.
Embora a entropia represente a distribuição do uso do solo no setor censitário, não estavam disponíveis informações sobre o uso de pisos acima do nível térreo, como no caso dos edifícios. Por essa razão, o indicador de densidade comercial foi incluído em adição à entropia. A densidade comercial foi estimada a partir dos cadastros de licenças para uso comercial (> 96000 licenças). Para o cálculo, determinou-se a localização espacial do estabelecimento na cidade (coordenadas $\times$ e y). Em seguida, obteve-se o indicador de densidade comercial pela razão entre o número de licenças comerciais por área em cada setor censitário.

Finalmente, o indicador de walkability foi obtido pela soma dos indicadores parciais (entropia, densidade comercial, densidade residencial e conectividade de ruas), convertidos em unidade de desvio-padrão por meio do escore $Z$. Todos os setores censitários da cidade ( $\mathrm{n}=2$ 125) foram incluídos, e para cada setor censitário foi calculado o escore de walkability. A figura 1 apresenta grafica-

FIGURA 1. Exemplos dos indicadores de intersecção das ruas, uso diversificado do solo densidade comercial em setores censitários de alto e baixo walkability
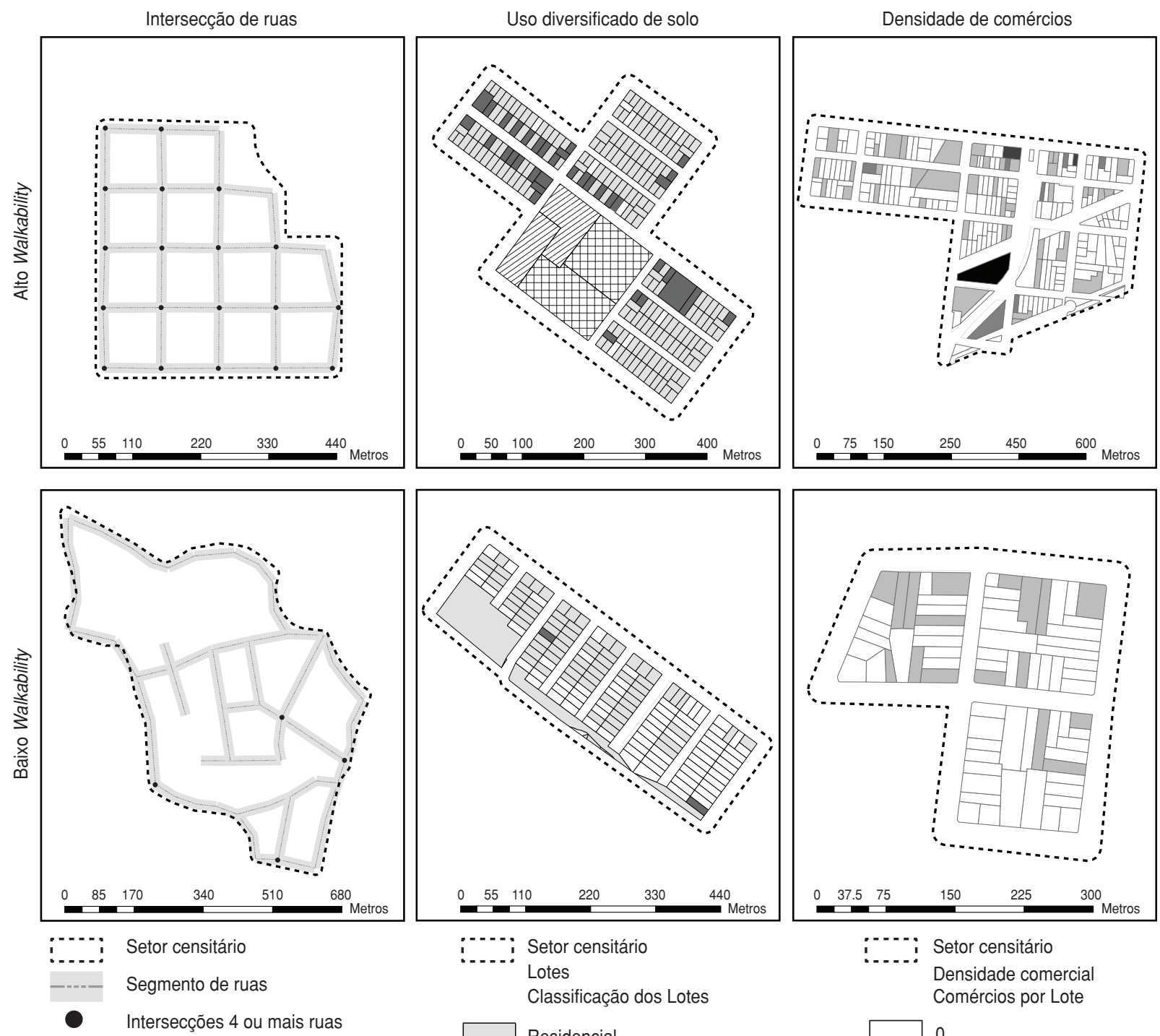
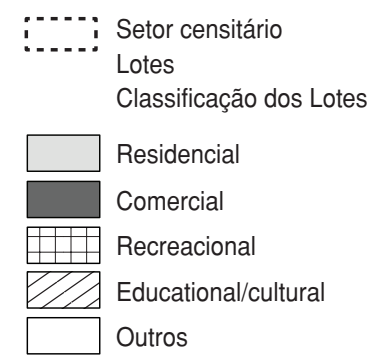

Residencial

Comercial

Recreacional

Educational/cultural

Outros

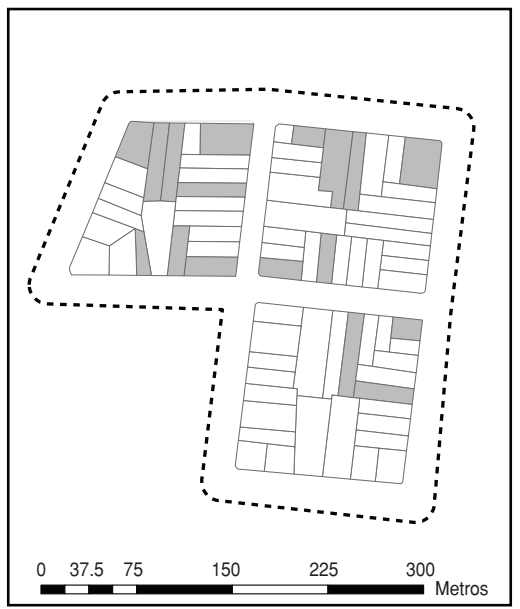

:- Setor censitário Densidade comercial Comércios por Lote

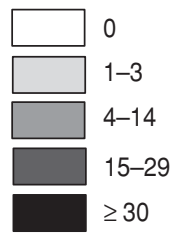


mente um exemplo das variáveis densidade de intersecção de ruas, uso do solo e densidade comercial e do conceito de alto e baixo walkability.

\section{Seleção dos setores censitários}

Os decis de walkability dos setores censitários foram computados. Os setores no $8^{\circ}$ e $9^{\circ}$ decis foram considerados de alto walkability, enquanto os localizados no $2^{\circ}$ e $3^{\circ}$ decis foram classificados como de baixo walkability. O mesmo procedimento foi adotado para a classificação da renda, sendo os setores classificados em renda elevada ( $8^{\circ}$ e $9^{\circ}$ decis) ou baixa $\left(2^{\circ}\right.$ e $3^{\circ}$ decis), considerando a renda média dos responsáveis pelos domicílios contidos em cada setor.

Finalmente, os setores de alto e baixo walkability e alta e baixa renda foram considerados elegíveis para o estudo. Foram excluídos os setores sem domicílios, compostos por uma ou duas quadras e os setores adjacentes com categorias extremamente distintas de renda e walkability (por exemplo, um setor de alto walkability localizado ao lado de um setor de baixo walkability). Nesse caso, ambos os setores eram excluídos. Ao todo, foram selecionados 16 setores de alto walkability e 16 de baixo walkability, sendo oito de renda baixa e oito de renda elevada em cada categoria (conforme protocolo do IPEN-Study, considerando o número mínimo de unidades amostrais para análise multinível).

\section{Seleção dos domicílios}

A seleção de domicílios considerou a amostra mínima requerida (500 indivíduos) em cada local participante do IPEN-Study. Esta estimativa considera a análise combinada de dados oriundos de todos os locais de estudo para determinar variância de AF explicada pelo ambiente construído $\left(\mathrm{R}^{2}\right)$. Para tanto, considerouse um poder $\geq 0,80(P<0,05)$ e variações entre 0,1 e 0,5 pontos de mudança no $\mathrm{R}^{2}$ da $\mathrm{AF}$ entre diferentes setores. Neste estudo, adotou-se como amostra mínima 22 indivíduos (50\% mulheres) por setor censitário, totalizando 704 pessoas, considerando uma distribuição equitativa entre os gêneros. Essa sobre-amostragem foi estabelecida para aumentar o poder das análises multivariadas.

Para seleção dos domicílios, os 32 setores foram visitados e os domicílios ar- rolados. Foram identificadas 10063 residências (média de $314 \pm 111$ domicílios / setor). Em seguida foi elaborada uma lista com o número de domicílios/setor e determinado o intervalo de seleção (razão entre o número total e o número mínimo de domicílios por setor censitário). Em cada setor, foram sorteados de maneira sistemática 22 domicílios, sendo 176 domicílios por estrato de walkability e renda e 704 domicílios no total.

\section{Seleção e entrevistas dos participantes}

Em cada domicílio sorteado, com base no número de moradores elegíveis, listados em ordem decrescente de idade, foi selecionado um indivíduo de forma aleatória. Foram elegíveis adultos de ambos os sexos, com idade entre 18 e 65 anos, residentes no setor censitário por ao menos 1 ano, considerando a data da coleta de dados. Indivíduos que não residiam no domicílio sorteado (como empregados domésticos e visitantes), aqueles com alguma limitação física que impedisse a prática de $\mathrm{AF}$ e ainda aqueles com limitações cognitivas que impedissem a compreensão das questões do questionário foram excluídos do estudo. Caso o indivíduo sorteado não estivesse na residência no momento da visita, ou não pudesse responder ao inquérito naquele momento, foi agendado um segundo encontro com o sujeito. Em caso de recusa, a residência à direita foi automaticamente selecionada. Antes de considerar uma recusa foram realizadas três tentativas de contato com o indivíduo sorteado.

\section{Instrumentos de pesquisa}

O IPEN-Study combina o uso de medidas autorrelatadas (questionários) e objetivas. Para tanto, foram utilizadas medidas autorrelatadas de ambiente e AF previamente validadas para a população brasileira, sendo as principais a Abbreviated Neighborhood Walkability Scale (A-NEWS) (20), que avalia a percepção do ambiente na comunidade, e o International Physical Activity Questionnaire (IPAQ)(21).

\section{Coleta de dados de AF com acelerômetros}

A AF foi mensurada de maneira objetiva através do uso de acelerômetro (modelos 7164 e GT1M), instrumento válido e fidedigno para emprego em adultos (22). Adotou-se a seguinte classificação para as intensidades de AF: a) atividades de intensidade leve (101 a 1952 counts/min); b) moderadas (1 953 a 5724 counts $/ \mathrm{min}$ ) e c) vigorosas ( $\geq 5724$ counts/min) (23).

Os dados foram analisados através dos softwares Actigraph 4.4.1, para programação do aparelho e Meter Plus 4.2 para tratamento dos dados. As informações foram consideradas válidas quando: a) o indivíduo utilizou o aparelho por ao menos 5 dias; b) apresentou $\geq 10$ horas válidas/dia; e c) horas de uso que registrassem menos de 60 zeros consecutivos (19).

A coleta de dados com acelerômetros durou aproximadamente 11 dias por sujeito, compreendendo: a) dia 1, programação do aparelho e entrega ao entrevistador; b) dia 2, entrega do acelerômetro ao participante e explicação dos procedimentos de utilização; c) do dia 3 ao 9, período de uso do aparelho; d) dia 10, retirada do acelerômetro; e d) dia 11, download e conferência dos dados.

$\mathrm{O}$ controle de qualidade quanto ao uso do equipamento foi realizado, por ligação telefônica, no segundo e quinto dia de uso do aparelho. Um protocolo foi adotado para verificar o uso do aparelho e para resolver dúvidas. Cada participante tinha à disposição um número de telefone e um endereço eletrônico para acesso em caso de dúvidas.

Estabeleceu-se que ao menos $50 \%$ da amostra do inquérito $(n=302)$ seriam elegíveis para o uso do acelerômetro, considerando a distribuição de sexo e setores censitários. No entanto, considerando a relativa escassez de informação sobre a taxa de resposta para o uso de acelerômetros em inquéritos domiciliares no Brasil, optou-se por enviar aparelhos a 381 sujeitos (excesso $\approx 25 \%$ ).

O estudo foi aprovado pelo Comitê de Ética em Pesquisa da Pontifícia Universidade Católica do Paraná (PUCPR) (protocolo $\left.\mathrm{n}^{\circ} 3034 / 001 / 1\right)$ e os dados foram coletados entre agosto e dezembro de 2010. Todos os participantes assinaram o termo de consentimento livre e esclarecido.

\section{RESULTADOS}

Ao todo foram incluídos 16 setores de alto walkability e 16 de baixo walkability, 
TABELA 1. Estatística descritiva dos indicadores que compuseram o escore de walkability ${ }^{\mathrm{a}}$ e renda média no setor censitário

\begin{tabular}{|c|c|c|c|c|c|}
\hline Estrato de walkability e renda & $\begin{array}{l}\text { Densidade residencial } \\
\left(\text { domicílio } / \mathrm{km}^{2}\right)\end{array}$ & $\begin{array}{l}\text { Densidade de intersecções } \\
\text { de ruas (intersecções } / \mathrm{km}^{2} \text { ) }\end{array}$ & $\begin{array}{l}\text { Uso diversificado do solo } \\
\text { (entropia })^{\mathrm{b}}\end{array}$ & $\begin{array}{l}\text { Densidade comercial } \\
\text { (estabelecimentos } / \mathrm{km}^{2} \text { ) }\end{array}$ & Renda média do setor $(\mathrm{R} \$)$ \\
\hline \multicolumn{6}{|l|}{ Alto e alta $(n=8)$} \\
\hline Média & 3572,80 & 119,10 & 0,50 & 2040,74 & 2252,00 \\
\hline (Mínimo-máximo) & (1 105,8-7 043,3) & $(60,2-189,6)$ & $(0,4-0,7)$ & $(522,8-5724,1)$ & $(1605,7-2720,0)$ \\
\hline Média do escore $Z$ & 0,03 & 0,72 & 0,85 & 0,52 & 0,79 \\
\hline \multicolumn{6}{|l|}{ Alto e baixa $(n=8)$} \\
\hline Média & 3313,46 & 114,03 & 0,48 & 665,59 & 647,24 \\
\hline Média do escore $Z$ & $-0,02$ & 0,63 & 0,72 & $-0,11$ & $-0,63$ \\
\hline \multicolumn{6}{|l|}{ Baixo e alta $(n=8)$} \\
\hline Média & 1226,04 & 36,24 & 0,22 & 326,43 & 1936,40 \\
\hline Mediana & 1144,28 & 36,12 & 0,21 & 335,94 & 1848,55 \\
\hline (Mínimo-máximo) & $(295,5-2105,5)$ & $(8,3-84,1)$ & $(0,1-0,4)$ & $(192,6-435,8)$ & $\begin{array}{c}(1558,3- \\
2510,6)\end{array}$ \\
\hline Média Z-Score & $-0,38$ & $-0,69$ & $-0,67$ & $-0,27$ & 0,51 \\
\hline Média do escore $Z$ & $-0,08$ & $-0,50$ & $-0,97$ & $-0,29$ & $-0,67$ \\
\hline \multicolumn{6}{|l|}{ Todos $(n=2125)$} \\
\hline Média & 3418,88 & 76,94 & 0,34 & 911,80 & 1361,60 \\
\hline Mediana & 2159,31 & 69,35 & 0,36 & 380,40 & 958,23 \\
\hline (Mínimo-máximo) & $(0,0-79677,3)$ & $(0,0-483,2)$ & $(0,0-0,9)$ & $(0,0-35858,5)$ & $(0,0-11289,6)$ \\
\hline Média do escore $Z$ & 0,00 & 0,00 & 0,00 & 0,00 & 0,00 \\
\hline
\end{tabular}

a Soma dos escores $Z$ de densidade residencial, densidade de intersecções de ruas, uso misto do solo e densidade comercial.

${ }^{b}$ Entropia calculada para cinco categorias de uso de solo (residencial, comercial, espaços de lazer, educacional/cultural, outros). A entropia varia de 0 (predominância de apenas um tipo de uso do solo) a 1 (distribuição igual entre todas as categorias de uso).

TABELA 2. Descrição do número de domicílios arrolados, visitados, elegíveis e taxa de sucesso e recusas no processo de seleção e aplicação do projeto ESPAÇOS de Curitiba, Brasil, 2010

\begin{tabular}{|c|c|c|c|c|c|c|}
\hline \multirow[b]{2}{*}{$\begin{array}{c}\text { Estrato de } \\
\text { walkability e renda }\end{array}$} & \multicolumn{3}{|c|}{ Domicílios } & \multicolumn{3}{|c|}{ Participantes } \\
\hline & Arrolados & Visitados & $\begin{array}{c}\text { Elegíveis }^{a} \\
\text { No. }(\%)\end{array}$ & $\begin{array}{l}\text { Sucesso } \\
\text { No. (\%) }\end{array}$ & $\begin{array}{l}\text { Recusas }^{b} \\
\text { No. }(\%)\end{array}$ & $\begin{array}{l}\text { Visitas/domicílio } \\
\text { ( } \pm \text { desvio-padrão) }\end{array}$ \\
\hline Alto e alta & 2640 & 280 & $243(86,8)$ & $173(71,2)$ & $60(24,7)$ & $1,82( \pm 0,82)$ \\
\hline Alto e baixa & 1912 & 330 & $286(86,7)$ & $179(62,6)$ & $96(33,6)$ & $1,53( \pm 0,77)$ \\
\hline Baixo e alta & 3309 & 283 & $247(87,3)$ & $175(70,9)$ & $71(28,7)$ & $1,76( \pm 0,86)$ \\
\hline Baixo e baixa & 2202 & 308 & $276(89,6)$ & $172(62,3)$ & $83(30,1)$ & $1,51( \pm 0,77)$ \\
\hline Total & 10063 & 1201 & $1052(87,6)$ & $699(66,4)$ & $310(29,5)$ & $1,65( \pm 0,81)$ \\
\hline
\end{tabular}

a Valores calculados com base no número de domicílios visitados.

b Valores calculados com base no número de domicílios elegíveis.

sendo oito de baixa renda e oito de renda elevada em cada categoria. A tabela 1 apresenta a descrição dos indicadores que compuseram o escore de walkability e a renda dos setores que compuseram os estratos de alto e baixo walkability.

A amostra final foi de 699 participantes $(53,1 \%$ mulheres). Foram visitados 1201 domicílios, sendo 12,4\% não elegíveis e $25,8 \%$ recusas (tabela 2). Em $3,5 \%$ dos casos, embora efetivados a seleção e o agendamento, a entrevista não foi concluída por indisponibilidade de tempo do entrevistado. A proporção final de participantes em relação ao número de domicílios elegíveis (taxa de sucesso) foi de 66,4\%. A taxa de sucesso foi mais elevada nos estratos de alta renda $(71,2 \%$ e $70,9 \%)$ em comparação aos de renda baixa $(62,6 \%$ e $62,3 \%$; $P=$ $0,034)$, enquanto a taxa de recusas geral foi $29,5 \%$, valor similar entre os estratos $(P=0,165)$. Cada domicílio foi visitado em média $1,65( \pm 0,81)$ vez, sendo significativamente maior o número de visitas nos estratos de renda alta quando comparados aos de renda baixa $(P<0,001)$. O tempo médio de entrevista foi de 46 minutos e 57 segundos ( \pm 16 minutos e 56 segundos).

Entre os recrutados, $96,3 \%(n=367)$ aceitaram utilizar o acelerômetro (figura
2). Após o recolhimento dos aparelhos, verificou-se que $80,3 \%(n=306)$ dos sujeitos fizeram uso correto. Os $62(16,3 \%)$ que apresentaram informações não válidas foram convidados a reutilizar o aparelho. Ao todo, 5,5\% $(\mathrm{n}=21)$ aceitaram o procedimento e retornaram informações válidas. No final, $327(85,8 \%)$ sujeitos utilizaram o acelerômetro com dados válidos. As perdas totalizaram 14,1\% ( $\mathrm{n}=$ 54), sendo $3,7 \%$ recusas, $4,2 \%$ recusas de reutilização, 1,3\% acelerômetros com dados não válidos na reutilização e 4,9\% de acelerômetros não entregues aos participantes dentro do prazo previamente determinado no estudo. 
FIGURA 2. Representação do número de acelerômetros enviados, utilizados, reutilizados e recusas no processo de seleção e aplicação do projeto ESPAÇOS de Curitiba, Brasil, 2010

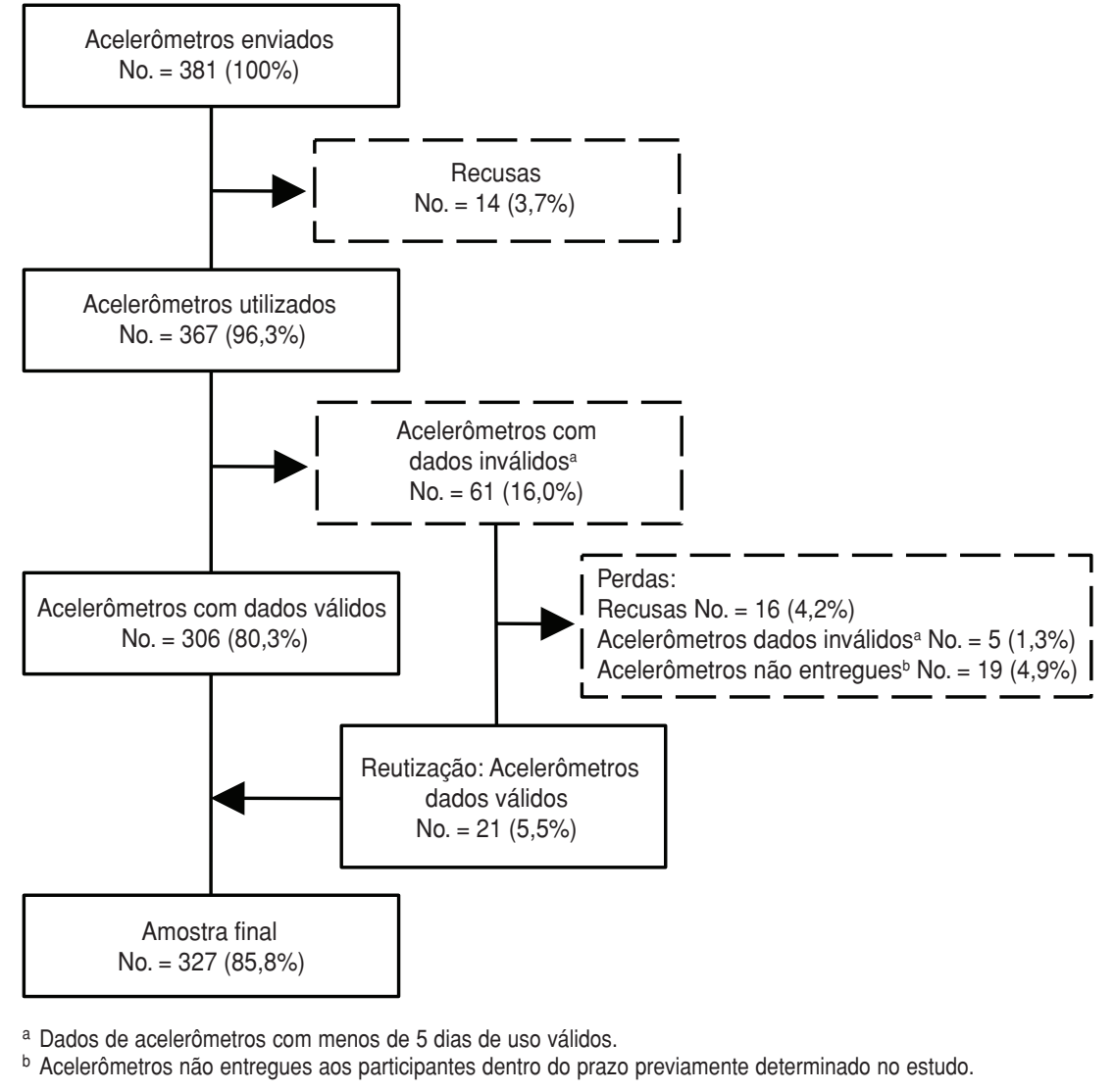

\section{DISCUSSÃO}

O projeto ESPAÇOS de Curitiba foi desenvolvido como parte do IPEN-Study na tentativa de aumentar a quantidade de informações sobre a relação entre ambiente construído e AF e ampliar a compreensão sobre essa relação em países com diferentes características socioculturais. Trata-se de um estudo inovador e, portanto, diversos desafios também se apresentam em seu desenvolvimento.

Uma das maiores dificuldades nos estudos que investigam de maneira objetiva a associação do ambiente construído com a AF é a disponibilidade dos dados baseados no sistema de informação geográfica (SIG). Neste estudo, os dados somente estavam disponíveis devido ao longo histórico de planejamento urbano de Curitiba e ao desenvolvimento de informações nesta área. Por essa razão, uma ampla base de informações estava disponível sem custo. Assim, o maior desafio foi desenvolver o domínio do uso de ferramentas e métodos usual- mente empregados na área de urbanismo, porém pouco aplicados no contexto da AF.

A viabilidade do emprego das informações foi demonstrada na medida em que os componentes do indicador de walkability empregados em outros países (uso misto do solo, densidade comercial, densidade residencial e conectividade das ruas) foram adequadamente reproduzidos. No entanto, algumas características se distinguem. Por exemplo, em países como Estados Unidos (19) e Austrália (17), o indicador de uso misto de solo considera a área de cada estabelecimento comercial. No contexto brasileiro, tal informação não está usualmente disponível nas bases de dados, uma vez que está associada com informações relativas à tributação, que no Brasil são sigilosas. Todavia, mesmo sem considerar essa característica, foi possível classificar e identificar áreas com maior ou menor walkability.

No inquérito, a taxa de sucesso foi de $66,4 \%$, sendo maior nos estratos de renda elevada. A taxa de recusa foi de $29,5 \%$ e similar entre os estratos. Os valores apresentados são similares àqueles relatados em outras investigações, que reportam valores em torno de $60 \%$ entre os sujeitos elegíveis (24, 25). É importante notar que tais estudos usualmente oferecem incentivos aos participantes (como cupons de compra ou brindes, pagamento em dinheiro) como forma de incrementar a taxa de participação (19), procedimento que não é permitido em pesquisas realizadas no Brasil. Portanto, em nosso projeto, a taxa de sucesso pode ser considerada satisfatória em relação ao esperado para inquéritos domiciliares.

Em geral, menores taxas de sucesso têm sido relatadas em populações economicamente mais vulneráveis (26), o que também foi verificado em nosso estudo. Porém, a distinção entre os componentes da "não reposta" em um inquérito, nomeadamente taxa de sucesso e taxa de recusa, tem sido apontada como uma abordagem mais adequada para analisar os vieses de resposta (24). No presente estudo, para uma mesma taxa de resposta, o número de visitas foi significativamente maior nos estratos de alta renda quando comparados aos de renda baixa. Tal resultado não coincide com as evidências da literatura, que sugerem que o declínio nas taxas de resposta em inquéritos domiciliares parece mais relacionado à recusa do que propriamente à capacidade de encontrar os participantes (24). Por outro lado, o tipo de domicílio (casa ou apartamento) pode afetar essas taxas, com menores taxas de sucesso em apartamentos (24). Em nosso estudo, assim como em muitas capitais brasileiras, as áreas de renda elevada são predominantemente compostas por edifícios residenciais, o que pode explicar em parte os achados aqui relatados.

Ao final da coleta de dados, 85,8\% dos participantes recrutados fizeram uso correto dos acelerômetros. Este resultado é ligeiramente inferior ao encontrado na Bélgica (25), porém acima do relatado nos Estados Unidos $(64,6 \%)$ (27). Todavia, as diferentes características metodológicas limitam tais comparações. Na Bélgica (25) foram considerados válidos os dados de 4 dias ou mais compostos de horas "válidas" com até 59 zeros consecutivos de registro, critério menos restritivo do que o empregado em nosso estudo. Por outro lado, 
o estudo americano utilizou o correio como meio de entrega e recolhimento dos aparelhos. Tais características sugerem que a taxa de resposta com dados "válidos" em nosso estudo foi elevada quando comparada à de pesquisas similares. Alguns procedimentos empregados podem ajudar a compreender esses resultados. Por exemplo, o uso de uma identidade própria, com uma mensagem de fácil assimilação, pode ter auxiliado o contato com os participantes. De fato, há evidências sugerindo que o emprego de mensagens que apresentem significado para a população aumenta a prontidão para o uso de informações de saúde (28). Ainda, o controle de qualidade envolveu o envio de cartas de apresentação e telefonemas, estratégias que são sugeridas em inquéritos domiciliares.

Algumas limitações da metodologia do IPEN-Study devem ser consideradas. No caso específico de Curitiba, os dados oriundos do SIG referiam-se ao mesmo período da coleta de dados (2010). Contudo, deve-se levar em conta que tais dados não foram originalmente obtidos para estudos sobre AF, e podem conter erros difíceis de serem detectados. Ainda, a reaplicação desse tipo de es- tudo em outras cidades está condicionada à disponibilidade de informações desse tipo. Nos dados apresentados sobre o presente estudo, deve-se considerar ainda que as análises sobre as taxas de resposta consideraram apenas dados agregados e não características individuais (como sexo e idade).

Futuros estudos que pretendam investigar a relação entre ambiente e atividade física, particularmente na América Latina, deverão considerar aspectos relacionados ao acesso de dados, capacidades técnicas e características sociais de cada localidade. Tais aspectos devem ser incorporados no planejamento de pesquisa e no processo de controle de qualidade para garantir a otimização de recursos e adequadas taxas de resposta.

\section{Conclusão}

O presente artigo descreveu os métodos empregados em um estudo para avaliar a relação entre ambiente construído e AF. Métodos mistos envolvendo o uso de medidas objetivas para avaliar a AF e o ambiente construído, empregados em países desenvolvidos, apresentaram aplicabilidade no contexto brasileiro. Os desafios encontrados no presente estudo relacionaram-se ao acesso a informações georreferenciadas, conhecimento acerca das técnicas de tratamento e análise de dados especializados e ainda controle de qualidade, particularmente no que tange ao emprego de acelerômetros.

Agradecimentos. O IPEN-Study obteve em 2009 financiamento dos National Institutes of Health (NIH), Estados Unidos, sob a liderança do Dr. James F. Sallis, da San Diego State University. Os autores AAFH e PBG possuíam bolsa CAPES-REUNI no período do estudo. No Brasil, o estudo foi coordenado pelo Grupo de Pesquisa em Atividade Física e Qualidade de Vida (GPAQ) da Pontifícia Universidade Católica do Paraná e realizado em colaboração com a Universidade Federal do Paraná, a Universidade Federal de Pelotas e a Universidade de São Paulo. Os autores agradecem aos membros do GPAQ pelo esforço no desenvolvimento do estudo, assim como aos profissionais do Instituto de Planejamento e Pesquisa Urbano de Curitiba pelo profissionalismo e boa vontade e pela disponibilização dos dados.

\section{REFERÊNCIAS}

1. Rodgers A, Ezzati M, Vander Hoorn S, Lopez $A D$, Lin RB, Murray CJ, et al. Distribution of major health risks: findings from the Global Burden of Disease study. PLoS Med. 2004;1(1):e27.

2. Cervero R, Sarmiento OL, Jacoby E, Gomez LF, Neiman A. Influences of built environments on walking and cycling: lessons from Bogotá. Int J Sust Transp. 2009;3(4):37-41.

3. Hino AA, Reis RS, Sarmiento OL, Parra DC, Brownson RC. The built environment and recreational physical activity among adults in Curitiba, Brazil. Prev Med. 2011;52(6):419-22.

4. Allender S, Foster C, Hutchinson L, Arambepola C. Quantification of urbanization in relation to chronic diseases in developing countries: a systematic review. J Urban Health. 2008;85(6):938-51.

5. Hino AAF, Reis RS, Florindo AA. Built environment and physical activity: a brief review of evaluation methods. Rev Bras Cineantropom Desempenho Hum. 2010;12(5):387-94.

6. Gebel K, Bauman AE, Petticrew M. The physical environment and physical activity: a critical appraisal of review articles. Am J Prev Med. 2007;32(5):361-9.

7. Saelens BE, Handy SL. Built environment correlates of walking: a review. Med Sci Sports Exerc. 2008;40(7 Suppl):S550-66.

8. Wendel-Vos W, Droomers M, Kremers S, Brug J, van Lenthe F. Potential environmental de- terminants of physical activity in adults: a systematic review. Obes Rev. 2007;8(5):425-40.

9. Sallis JF, Bowles HR, Bauman A, Ainsworth BE, Bull FC, Craig CL, et al. Neighborhood environments and physical activity among adults in 11 countries. Am J Prev Med. 2009;36(6):484-90

10. United Nations. Population Division, Department of Economic and Social Affairs United Nations Secretariat. World urbanization prospects: The 2001 revision, data tables and highlights. Nova Iorque: United Nations; 2002. Disponível em: http://www.google.com.br/ url? sa $=t \& r c t=j \& q=\&$ esrc $=s \&$ source $=w e b \&$ $\mathrm{cd}=1 \&$ ved $=0$ CCUQFjAA\&url $=\mathrm{http} \% 3 \mathrm{~A} \%$ 2F\%2Fwww.un.org\%2Fesa\%2Fpopulation \%2Fpublications\%2Fwup2001\%2Fwup2001 dh.pdf\&ei=KUBnUK_GBIHa8wSMpYHYC Q\&usg=AFQjCNGN3oViekp2xjJyCU-D3gnRNjQzQ\&cad=rja Acessado em setembro de 2012.

11. Reis RS, Hallal PC, Parra DC, Ribeiro IC, Brownson RC, Pratt M, et al. Promoting physical activity through community-wide policies and planning: findings from Curitiba, Brazil. J Phys Act Health. 2010;7 Suppl 2:S137-45.

12. IPPUC. Planejamento. História do planejamento em Curitiba. Curitiba: Instituto de Pesquisa e Planejamento Urbano de Curitiba; 2008. Disponível em: http://www.ippuc.org. br/ Acessado em 28 de setembro de 2012.
13. Hino AAF, Reis RS, Ribeiro IC, Parra DC, Brownson RC, Fermino RC. Using observational methods to evaluate public open spaces and physical activity in Brazil. J Phys Act Health. 2010;7 Suppl 2:S146-54.

14. Reis RS, Hino AA, Florindo AA, Añez CR, Domingues MR. Association between physical activity in parks and perceived environment: a study with adolescents. J Phys Act Health. 2009;6(4):503-9.

15. Gonçalves PB, Reis RS, Añez CRR, Florindo AA. Validade e fidedignidade de um instrumento para avaliar o ambiente doméstico relacionado à atividade física em idosas. Rev Bras Ativ Fis Saude. 2010;15(2):82-7.

16. Parra DC, Hoehner CM, Hallal PC, Ribeiro IC, Reis R, Brownson RC, et al. Perceived environmental correlates of physical activity for leisure and transportation in Curitiba, Brazil. Prev Med. 2011;52(3-4):324-8.

17. Sallis JF, Cervero RB, Ascher W, Henderson KA, Kraft MK, Kerr J. An ecological approach to creating active living communities. Annu Rev Public Health. 2006;27:297-322.

18. Owen N, Cerin E, Leslie E, duToit L, Coffee N, Frank LD, et al. Neighborhood walkability and the walking behavior of Australian adults. Am J Prev Med. 2007;33(5):387-95.

19. Sallis JF, Saelens BE, Frank LD, Conway TL, Slymen DJ, Cain KL, et al. Neighborhood built environment and income: examin- 
ing multiple health outcomes. Soc Sci Med. 2009;68(7):1285-93.

20. Malavasi LM, Duarte MFS, Both J, Reis RS. Escala de mobilidade ativa no ambiente comunitário - news Brasil: retradução e reprodutibilidade. Rev Bras Cineantropom Desempenho Hum. 2007;9(4):339-50.

21. Hallal PC, Gomez LF, Parra DC, Lobelo F, Mosquera J, Florindo AA, et al. Lessons learned after 10 years of IPAQ use in Brazil and Colombia. J Phys Act Health. 2010;7 Suppl 2:S259-64.

22. Welk GJ. Use of accelerometry-based activity monitors to assess physical activity. Em: Welk GJ, editor. Physical activity assessments for health-related research. Champaign: Human Kinetics; 2002. Pp. 125-41.
23. Freedson PS, Melanson E, Sirard J. Calibration of the Computer Science and Applications, Inc. accelerometer. Med Sci Sports Exerc. 1998;30(5):777-81.

24. Heerwegh D, Abts K, Loosveldt G. Minimizing survey refusal and noncontact rates: do our efforts pay off. Surv Res Methods. 2007;1(1):3-10.

25. Van Dyck D, Cardon G, Deforche B, Sallis JF, Owen N, De Bourdeaudhuij I. Neighborhood SES and walkability are related to physical activity behavior in Belgian adults. Prev Med. 2010;50 Suppl 1:S74-9.

26. Kiezebrink K, Crombie IK, Irvine L, Swanson V, Power K, Wrieden WL, et al. Strategies for achieving a high response rate in a home interview survey. BMC Med Res Methodol. 2009;9(1):46

27. Tucker JM, Welk GJ, Beyler NK. Physical activity in U.S.: adults compliance with the Physical Activity Guidelines for Americans. Am J Prev Med. 2011;40(4):454-61.

28. Kreuter MW, Wray RJ. Tailored and targeted health communication: strategies for enhancing information relevance. Am J Health Behav. 2003;27 Suppl 3):227-32.

Manuscrito recebido em 6 de fevereiro de 2011. Aceito em versão revisada em 25 de julho de 2012.

ABSTRACT Objective. To describe the methods employed to assess the built environment and physical activity (PA) as part of a multicenter international study, and to discuss the challenges faced to obtain the necessary data in the Brazilian context.

\section{Projeto ESPAÇOS de Curitiba, Brazil: applicability of mixed research methods and geo-referenced information in studies about physical activity and built environments}

Methods. In 2010 a household survey was conducted with adults aged from 20 to 65 years in the city of Curitiba, Brazil. The study involved the used of geo-referenced information to measure walkability in all 2125 census sectors in Curitiba. Census sectors were categorized by walkability and income, taking into consideration the average income of heads of the family in each sector. Physical activity was assessed by selfreport and using an objective measure (accelerometers).

Results. Sixteen high walkability and 16 low walkability sectors were studied, with eight high-income and eight low-income sectors in each category. A total of 699 subjects were interviewed and 381 wore accelerometers. The response rate was $66.4 \%$ for the interviews and the compliance with accelerometer use was $85.8 \%(n=327)$.

Conclusions. The results show that it is feasible to conduct high-quality studies on physical activity and built environment in the Brazilian context in accordance with international standards.

Key words Motor activity; environment design; research design; Brazil. 